\title{
Components of the dorsal-ventral pathway also contribute to anterior-posterior patterning in honeybee embryos (Apis mellifera)
}

Megan J Wilson ${ }^{2 *}$, Nathan J Kenny ${ }^{1,3}$ and Peter K Dearden ${ }^{1}$

\begin{abstract}
Background: A key early step in embryogenesis is the establishment of the major body axes; the dorsal-ventral (DV) and anterior-posterior (AP) axes. Determination of these axes in some insects requires the function of different sets of signalling pathways for each axis. Patterning across the DV axis requires interaction between the Toll and Dpp/TGF- $\beta$ pathways, whereas patterning across the AP axis requires gradients of bicoid/orthodenticle proteins and the actions of a hierarchy of gene transcription factors. We examined the expression and function of Toll and Dpp signalling during honeybee embryogenesis to assess to the role of these genes in DV patterning.

Results: Pathway components that are required for dorsal specification in Drosophila are expressed in an AP-restricted pattern in the honeybee embryo, including Dpp and its receptor Tkv. Components of the Toll pathway are expressed in a more conserved pattern along the ventral axis of the embryo. Late-stage embryos from RNA interference (RNAi) knockdown of Toll and Dpp pathways had both DV and AP patterning defects, confirmed by staining with Am-sna, Am-zen, Am-eve, and Am-twi at earlier stages. We also identified two orthologues of dorsal in the honeybee genome, with one being expressed during embryogenesis and having a minor role in axis patterning, as determined by RNAi and the other expressed during oogenesis.
\end{abstract}

Conclusions: We found that early acting pathways (Toll and Dpp) are involved not only in DV patterning but also AP patterning in honeybee embryogenesis. Changes to the expression patterns and function of these genes may reflect evolutionary changes in the placement of the extra-embryonic membranes during embryogenesis with respect to the AP and DV axes.

Keywords: Anterior posterior, Apis mellifera, Axis formation, Dorsal ventral, Dpp, Evolution, Honeybee, Toll

\section{Background}

A critical early step in establishing the future body plan of any animal is the formation of the major body axes, the anterior-posterior axis (AP), and the dorsal-ventral axis (DV). Determination of these axes is established by an initial, often maternally driven, symmetry-breaking event, and as the process continues it requires precise zygotic control of gene expression across the body axes via cell-signalling pathways. DV patterning has been well studied in Drosophila melanogaster, which has a derived

\footnotetext{
* Correspondence: meganj.wilson@otago.ac.nz

${ }^{2}$ Developmental Biology Laboratory, Department of Anatomy, University of Otago, P.O. Box 56, Dunedin 9054, New Zealand

Full list of author information is available at the end of the article
}

mode of development compared to other insects [1,2]. One unique innovation present in the DV axis patterning mechanism of Cyclorraphan flies such as Drosophila is the fusion of the extraembryonic membranes, which arise as a single tissue, the amnioserosa [3]. In the majority of insects, including other Dipterans, these tissues arise separately [2-6]. A conserved requirement in serosa patterning in many insects is the expression of zenknüllt (zen), a Hox3 gene that has been co-opted, during insect evolution, into defining the serosal membrane $[3,4,7,8]$. In some insects, such as the honeybee, this expression also delineates where the amnion develops, and it has been suggested that it defines the border between the extraembryonic tissue and embryo [4].

Patterning of the dorsal epidermis and refinement of zen expression in Drosophila embryos, requires the 
expression of decapentaplegic (Dpp) at the dorsal midline. $D p p$ is a member of the TGF- $\beta$ family of signalling molecules, which activate the MAD pathway on the dorsal midline of the blastoderm embryo in a gradient-like fashion. Short gastrulation (Sog) acts by binding to and inhibiting Dpp activity, thus promoting formation of the presumptive neuroectoderm. In dorsal regions, Tolloid (a dorsally restricted protein) cleaves Sog to release Dpp, enhancing its activity [9]. This limits Dpp activity to the dorsal side of the embryo, where high levels of signalling leads to up-regulation of genes required for the specification of dorsal ectoderm epidermis and amnioserosal fate [10-12], and allows, in ventral regions with low Dpp activity, neuroectoderm to form. Megaselia abdita, a basal Cyclorraphan fly, also requires Dpp and zen expression for development of distinct amnion and serosa tissues [3,5]. In the beetle Tribolium castaneum, Dpp expression mirrors where zen-1 mRNA is found, and where the serosa-embryo border is positioned [13], indicating that Dpp has a conserved role in DV patterning. Using the honeybee (Apis mellifera) model, we previously found that $D p p$ mRNA was maternally expressed and localized to the dorsal side of the oocyte, rather than the embryo, and the MAD pathway was activated in overlying follicle cells, implying that the Dpp-MAD pathway may pattern the DV axis of the oocyte, rather than the embryo [14].

DV axis formation is initiated in the oocyte prior to fertilization and oviposition long before localized expression of Dpp in D. melanogaster. The first symmetrybreaking event is movement of the oocyte nucleus to an anterior-dorsal location [15]. This results in the asymmetric localization of gurken mRNA, encoding a TGF- $\beta$ like ligand, which in turn activates the EGF receptor in the overlying follicle cells [16]. Expression of pipe (pip) is restricted to ventral follicle cells, triggering a ventral protease cascade that cleaves and activates the signalling molecule Spätzle (Spz) [17]. Shortly after egg laying, active Spz protein binds to the Toll receptor on the ventral side of the egg resulting in localized ventral activation of the Toll pathway [18]. While there is no gene encoding an orthologue of gurken in most other insect genomes [19-21], there is another TGF- $\beta$-like member that is predicted to perform a similar role in DV patterning. TGF$\beta$-like mRNAs are expressed maternally in Tribolium, Nasonia, and honeybee, but only localized to the anterior-dorsal side of the oocyte in a similar manner to gurken mRNA in Nasonia and the honeybee [14,21].

A key target of the Toll pathway is Cactus. Phosphorylation of Cactus results in its degradation on the ventral side of the embryo [22,23]. Cactus is an inhibitor of Dorsal, a DNA-binding rel/NF- $\mathrm{kB}$ protein family regulated by nuclear-cytoplasmic shuttling [24]. Cactus normally binds to Dorsal and sequesters it in the cytoplasm. As
Cactus is degraded, however, on the ventral side of the embryo, Dorsal is free to translocate to the nucleus to regulate the expression of genes required for mesoderm and neuroectoderm patterning. In both short and long germ-band insects, loss of Toll signalling has been observed to result in dorsalization of the embryo and loss of the Dorsal DV gradient [25]. There are some subtle differences in the DV patterning network between Tribolium and Drosophila as, in Tribolium, Tc-Dorsal activates transcription of Tc-cact to negatively regulate its own function [25].

There is also emerging evidence for 'cross-talk' between AP and DV pathways in some insect groups, coordinating positional information across the body axes. The homeodomain-containing gene orthodenticle (otd) is required for anterior patterning in long and short germ-band arthropods [7,26-28]. DV fates are organized along the AP axis in a short germ-band insect Tribolium and this requires Tc-otd [29]. Otd, in honeybee embryos, controls formation of the serosa via regulation of Amzen expression [7]. Additionally, the anterior patterning gene hunchback $(h b)$ specifies correct zen expression in dorsal regions of the honeybee embryo [7].

These differences in the regulatory networks that define DV fates, combined with the relatively conserved nature of the molecules involved, make DV patterning an interesting model system in which to study the evolution of developmental pathways. To further understand how this pathway has changed over the course of insect evolution we examined the expression of some of the key orthologues of DV patterning genes during development of the honeybee embryo. Honeybee embryos follow a long germ-band mode of development that may have evolved independently from long germ-band development in Drosophila [30]. Using RNAi knockdown and in situ hybridization, we demonstrate that, while patterning of the ventral neuroectoderm via snail and twist expression and their necessity in refining zen expression is conserved, early-acting components of DV patterning are expressed differently to other insects, and RNAi knockdown of those components results in defects across both AP and DV axes.

\section{Methods \\ Phylogenetics}

Dorsal homologues were identified by tBlastN searches of insect genomes [31]. Multiple alignments of putative dorsal proteins were carried out in ClustalX. The resulting multiple alignment was analyzed in MrBayes 3.1.2 under the WAG model with default priors [32]. The Monte Carlo Markov Chain search was run over 1,000,000 generations with trees sampled every 1,000 generations. The first 250,000 generations were discarded as 'burnin'. The final tree was displayed in Dendroscope [33]. 


\section{In situ hybridization}

PCR-amplified cDNA fragments for all genes of interest (primer sequences for each gene are given in Table 1) were cloned into the pBluescript vector using standard cloning methods [13] for use as in situ hybridization probes. Digoxygenin (DIG)-RNA probes were synthesized and used in in situ hybridization experiments as described previously [30,34]. Am-Dpp RNA probe cloning has been described elsewhere [14]. DAPI staining was performed as previously described [35].

\section{Honeybee RNA interference (RNAi)}

Targeted mRNA knockdown was performed as described by Wilson and Dearden [36,37]. Briefly, dsRNA was synthesized from cDNA fragments of Am-Toll, Am-Dpp, and $A m-G B 19066$ cloned into pLitmus38i (NEB) using the MEGAscript RNA kit (Ambion). dsRNA was injected at $2.5 \mathrm{mg} / \mathrm{mL}$ in reverse osmosis-purified $\mathrm{H}_{2} \mathrm{O}$ into freshly laid honeybee embryos. For each target, between 300-500 embryos were injected, with 15-20\% surviving the injection process. Injected embryos were incubated for $30 \mathrm{~h}$ (stage 4) or $48 \mathrm{~h}$ (stage 9) at $35^{\circ} \mathrm{C}$ (80\% humidity) before collection for in situ hybridization or DAPI staining.

\section{Results and discussion}

\section{Expression of early-acting dorsal-ventral components in} honeybee embryos

We examined the expression patterns of honeybee orthologues of components of the Drosophila dorsalventral pathway. The BMP/Dpp pathway is a cascade, conserved in evolution, involved in DV patterning in both invertebrates and vertebrates [38]. We have previously shown that $A m-D p p$ is expressed maternally and that its mRNA is localized to dorsal regions of the oocyte [14], implying a possible early role in DV patterning during oogenesis. During embryogenesis, Am-Dpp mRNA is restricted to cells in the posterior two-thirds of the embryo prior to gastrulation (Figure 1A, stage 4) and is then upregulated as two distinct anterior and posterior domains of cells by stage 5 (Figure 1B).

This expression pattern is unusual when compared with $D p p$ expression in the Diptera. In Drosophila, zygotic $D m-D p p$ mRNA is localised to dorsal regions of the syncytial blastoderm embryo and has a role in patterning the dorsal ectoderm and amnioserosa $[11,39]$. Two other members of the Dipteran suborder Brachycera, the scuttle fly (Megaselia abdita) and hoverfly (Episyrphus balteatus), have similar dorsal expression domains of $D p p$ $[5,40]$. The Nematocerans Clogmia albipunctata (moth midge) and Anopholes gambiae (mosquito) have a different pattern of $D p p$ expression; $C a-D p p$ is expressed at the anterior and posterior ends of the blastoderm embryo [38], while $A g-D p p$ mRNA is initially expressed as a broad medio-lateral band extending from the anterior to the posterior of the embryo corresponding to the presumptive amnion, but absent from the dorsal midline region where the presumptive serosa is patterned (as determined by $A g$-zen expression) [41]. Tribolium Dpp $(T c-D p p)$ is initially expressed throughout the blastoderm embryo, with higher expression at the anterior pole. $T c-D p p$ expression at the anterior pole is later lost and $T c-D p p$ mRNA is detected at the border between serosa and germ rudiment in a stripe along the AP axis [13]. In the locust Schistocerca gregaria, Sg-Dpp RNA is detected at the posterior of the germ band and head lobes at $15 \%$ development as well as in necklace cells

Table 1 List of oligonucleotide primers used in this study

\begin{tabular}{|c|c|c|}
\hline Target & F primer & R primer \\
\hline$\overline{A m-T w i}$ & CAGCTCTGGAATTCCTCATAGTG & CCACCTGCTTGTATCGCTG \\
\hline Am-sog & GCAGAATGTACGTTCGGCA & GGCACGATAACGAGATAATTGTC \\
\hline$A m-s p z$ & CGCAATTTGCCAGTTCCA & CTATCAGATTCAAAATCGATGTGAC \\
\hline Am-pipe & GAAGTTCGCCTTCAACCTG & CACGGTCCGCCTTAAGTACA \\
\hline Am-GB18032 & CGAAAGACAAACCATACAGACC & ATGTGTACCGGGACTGGCT \\
\hline Am-sim & CTITGGACGGTTCGTGTTI & GGTCAATTGTGACACGTTCG \\
\hline Am-NK2.2 & ACGATGTTCCGTGACATAC & СTCTCTTCGTCTTGTACCTGTG \\
\hline Am-GB19066 & TCTTCCGCCAATCAAAATTC & СTCTITAACGGCGAGGACAG \\
\hline Am-Toll & ATTCCATTCGTCCCCAAAC & CATTATTTGATAATAGTAACT \\
\hline Am-sna & TTCACCACCATCACCATTACC & CGGAATACGTGGAGTACGATTT \\
\hline Am-cactus & CACCTITACACTTGGCTGTATTG & TCAGGAAGTGGTTCTGGTATTG \\
\hline Am-pnr & GAAGGAGACGTTGACGAAGC & CGCCACTGGATTGGTTAGTT \\
\hline$A m-t k v$ & AAATACGGCCTGTGGATCAG & CACTITTTCGCCTCTCCATC \\
\hline$A m-t / d$ & AGAACGCTTATCGTGGCAAT & ACTGCTAGCACCCATGCTCT \\
\hline
\end{tabular}




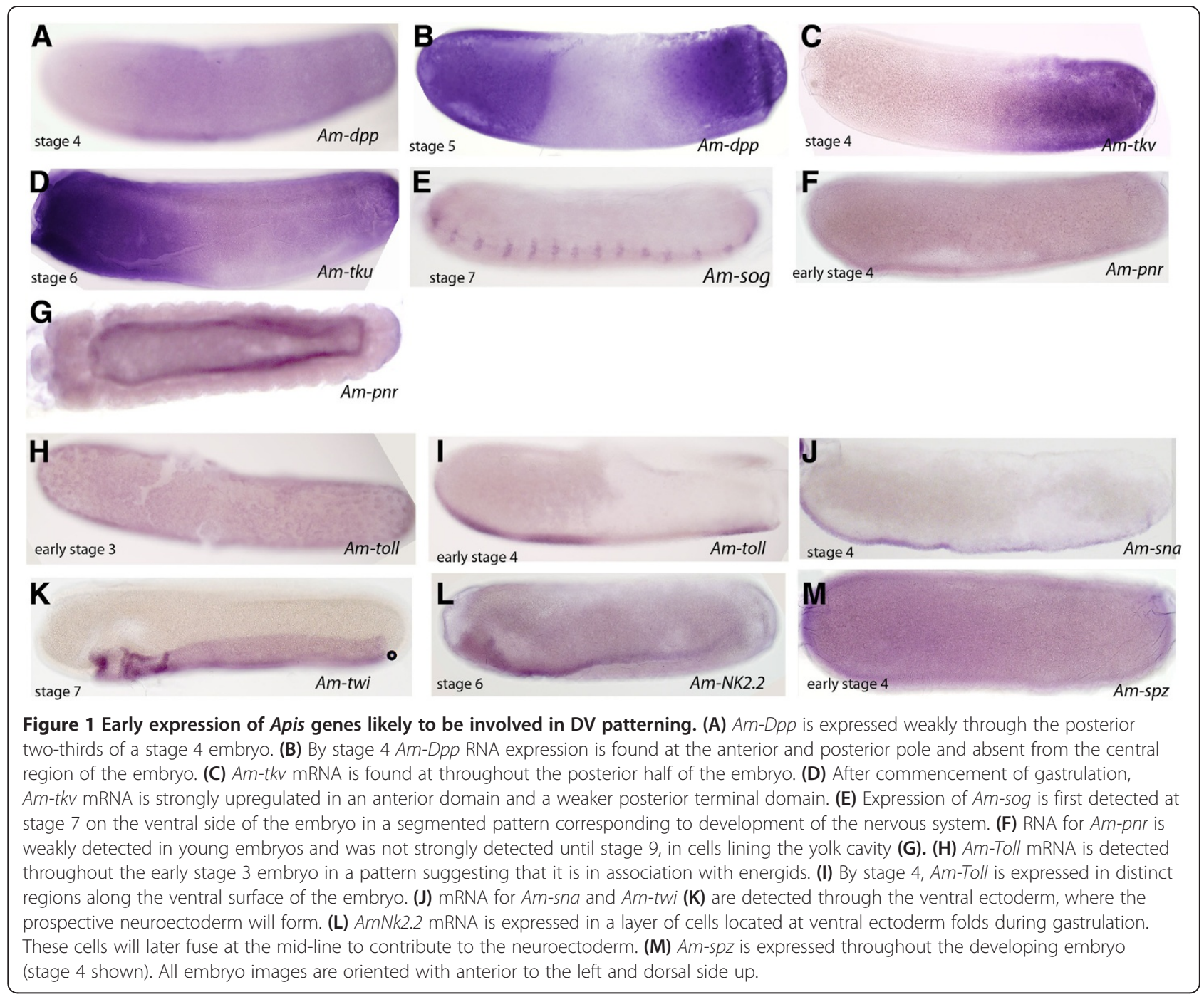

that include presumptive serosa cells [42]. These differences in the expression of Dpp seem to correlate with where the extra-embryonic membranes are positioned, particularly the way the serosa is determined, and whether it grows to surround the entire embryo or just a portion of the embryo.

To further understand the unusual observed expression pattern of $A m-D p p$, we also examined the expression of a Dpp receptor, thickveins (Am-tkv), in honeybee embryos. Knockdown of both maternal and zygotic $t k v$ in Drosophila produces a phenotype identical to loss of $D p p$ [43]. Am-tkv mRNA is detected initially throughout the posterior two-thirds of the blastoderm embryo (Figure 1C), followed by expression in the anterior and posterior domains of cells at stage 5 (Figure 1D), and is expressed in a similar pattern to that of $A m-D p p$ (Figure 1B). In contrast, in the dipteran Drosophila and Megaselia, tkv is initially ubiquitous and then becomes restricted to the dorsal side of the embryo, head mesoderm, and ventral ectoderm during cellularization [44]. A. gambiae tkv mRNA is expressed in an anterior cap of cells and dorsal-posterior end of the embryo and appears absent from the presumptive serosa [41]. This suggests that the expression pattern of the Tkv receptor may have co-evolved with regulation of Dpp expression.

Sog acts as an antagonist to the BMP/Dpp pathway by binding to, and trafficking, the Dpp protein [45]. Apis sog mRNA, however, is not detected in early embryos; instead, expression of Am-sog is first detected after gastrulation, in short stripes of cells across the ventral surface in presumptive neurectoderm (Figure 1E). In contrast, Tribolium sog mRNA, which is expressed in the blastoderm in a ventral stripe on the embryo, acts to control Dpp movement [46]. In Drosophila, sog has an essential transport role that shapes the Dpp gradient; while more ventrolaterally, Dpp inhibition is augmented by a transcriptional repressor, Brinker, to promote ventral neuroectoderm development [47]. No orthologue of 
sog has been identified in Nasonia genome or transcriptome sequences [48]; however, potential orthologues of brinker are found in both honeybee and Nasonia genomes (accession numbers XP_003249310.1 and NV 24459-PA respectively; unpublished data).

Pannier mRNA, encoding a zinc finger transcription factor, is upregulated in dorsal regions of the embryo by Dpp signalling in Drosophila. Am-pnr is not expressed during early to mid-stages of embryogenesis (Figure 1F), and it is not until the late stages that expression of Am-pnr is detected on ventral edges of the amnion (Figure 1G).

Am-Toll mRNA is expressed maternally and is found in association with energids throughout stage 1 embryos (Figure 1H). Just prior to gastrulation, Am-Toll mRNA becomes restricted to cells at the ventral surface of the embryo (Figure 1I), a pattern typical of the expression of Toll orthologues. We also examined the expression of factors that act downstream of the Toll pathway in Drosophila to pattern the mesoderm and neuroectoderm Am-sna, Am-twi, and Am-Nk2.2. These mRNAs are detected in cells on the ventral side of the embryo (Figure 1J-L) and later in the nervous system (data not shown) a typical expression pattern for these genes. The Apis Toll ligand spätzle (Am-spz) mRNA is detected throughout honeybee embryonic ectoderm (Figure 1M). This differs from Drosophila, where maternal spz transcripts are found throughout the syncytial blastoderm, but no zygotic expression of Dm-spz is detected until late stages in the gut [18].

A key component of the ventral pathway is Pipe. Restriction of Dm-Pipe to the ventral follicle cells in Drosophila egg chambers results in local activation of proteinases required for activation of the Toll ligand, spz. Apis Pipe RNA is not expressed during oogenesis (Figure 2A), and not detected until stage 6 in the posterior half of the embryo (Figure 2B). This expression pattern is different to that observed in Drosophila, where Dm-Pipe is maternally provided and becomes restricted to the ventral side of the oocyte (in follicle cells) [49].

Nuclear entry of Dorsal protein is regulated by Cactus, a IкB homolog, in both Drosophila and Tribolium [24,25]. Am-cact RNA is expressed maternally (Figure 2C,D), becoming enriched to the dorsal side of the oocyte during mid-oogenesis (Figure 2C) and then towards the anterior pole just before fertilization and laying (Figure 2D). In blastoderm-stage honeybee embryos, Am-cact RNA is present in the anterior half, with weak staining at the posterior pole (Figure 2E). This embryonic expression pattern is unusual; in Drosophila and Tribolium, Cactus is expressed only in a ventral stripe in the blastoderm embryo, and only the maternal contribution of Cactus has been ascribed a function [25]. In Nasonia, however, Nv-cact is initially expressed as a ventral stripe but prior to gastrulation only a terminal 'spot' of expression remains [48].
BLAST searches revealed the presence of two possible Dorsal orthologues in the honeybee genome. Rel/NF-кB proteins (such as Dorsal) all contain a rel homology domain required for DNA binding and dimerization [50]. They can be split into two classes: Class 1 includes the Relish proteins that have ankryin repeats at the C-terminus, while Class 2 includes Dorsal-like proteins that have a transactivation domain and act as transcriptional activators. We carried out phylogenetic analysis of the Dorsal/rel group of proteins from insects, including Dorsal and Dorsal-related immunity factor (Dif) proteins. Dipteran and coleopteran genomes encode two Dorsal-like proteins (Figure 2F), while hymenopteran (Nasonia, Apis mellifera, Bombus, and A. cephalotes (leaf cutter ant)) genomes contain between two to three Dorsal-like genes (Figure 2F).

Since neither Dorsal-like honeybee protein could be easily distinguished as being more 'Dorsal-like' based solely on sequence (Figure 2F), we examined the maternal and embryonic expression of both Apis Dorsallike genes, GB19066 and GB18032. Am-GB19066 mRNA is maternally expressed and detected throughout the oocyte from mid-oogenesis (Figure 2G). During embryogenesis, expression is detected throughout the ectoderm of the developing embryo with enrichment at the ventral and AP termini (Figure $2 \mathrm{H}$, stage 4). Am-GB18032 expression could only be detected in a subset of nurse cells at the posterior end of the nurse cell cluster in queen ovarioles (Figure 2I). No expression of Am-GB18032 was detected in oocytes or embryos (Figure 2I, data not shown). Since Drosophila and Tribolium Dorsal mRNAs are expressed throughout the embryo [51], we proposed Am-GB19066 as the most likely functional orthologue of Dorsal in honeybee, and undertook further analysis of this gene by dsRNA knockdown (Figure 3G,H).

\section{RNAi knockdown of Am-Toll, Am-GB19066 (Dorsal), and Am-Dpp}

Despite widespread conservation of Drosophila DV patterning genes between insect orders, the Apis orthologues of these genes have divergent patterns of expression compared to other insects, particularly Diptera. We examined the function of three key DV genes by dsRNA knockdown, and assessed the phenotypes produced in 48 hour embryos, a time at which morphological markers of DV patterning are present, using DAPI staining (Figure $3 \mathrm{~A}, \mathrm{~B}$, ventral view).

Targeted knockdown of Am-Toll by dsRNAi (AmToll $^{\mathrm{RNAi}}$ ) results in severe anterior defects and disrupted patterning along both AP and DV axes. Thoracic and anterior abdominal segments are fused and, while tracheal pits are visible, the segments are indistinguishable (indicated in Figures $3 \mathrm{C}$ and 1D). At the anterior end of the 


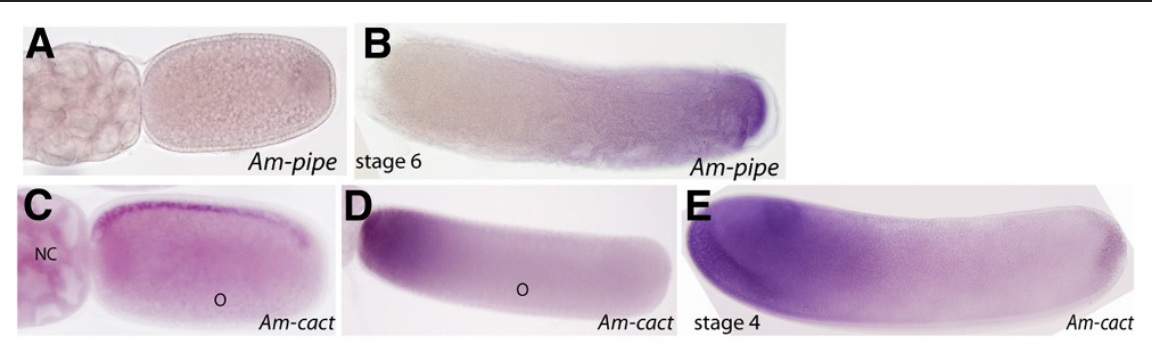

$\mathbf{F}$
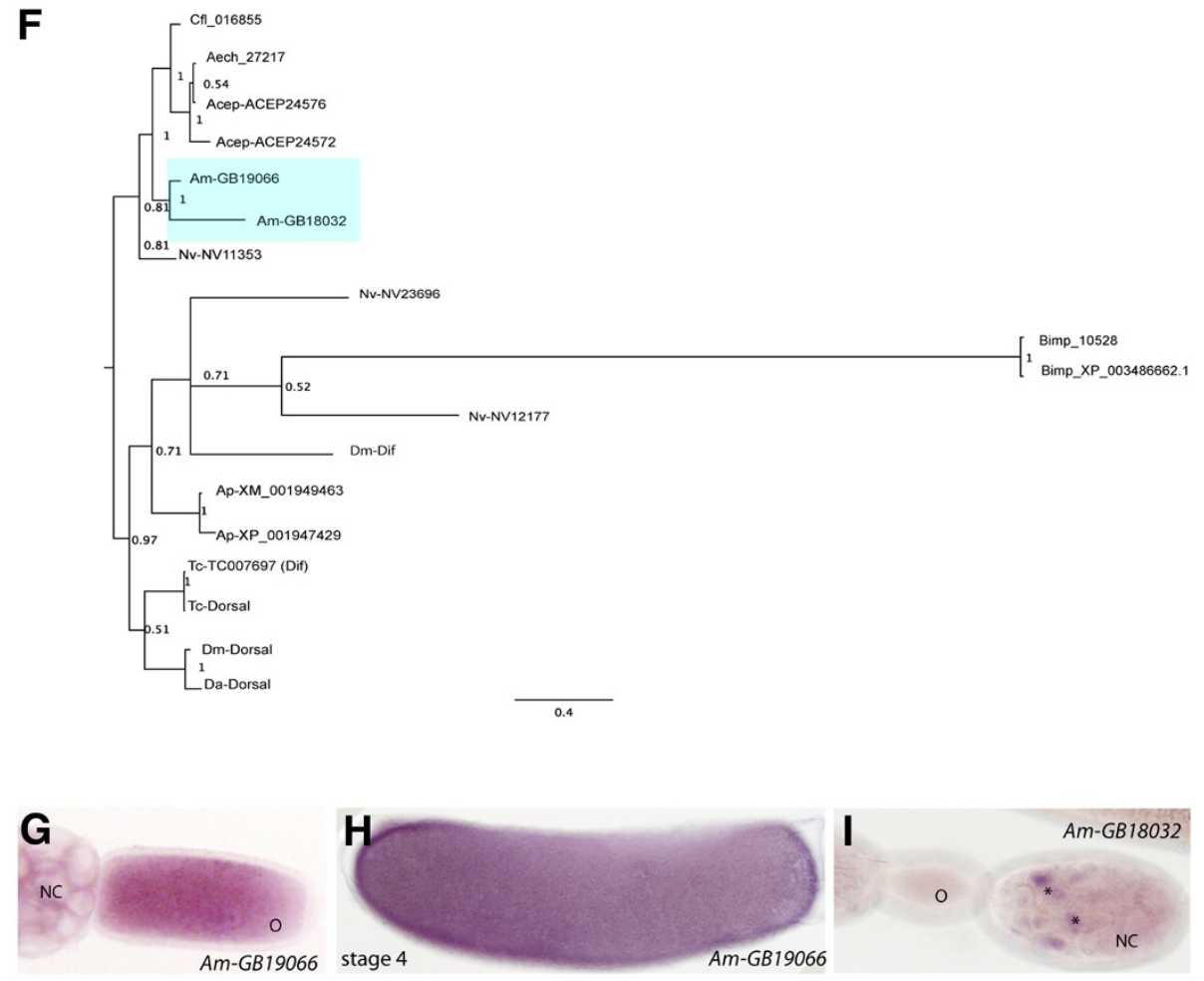

Figure 2 Phylogenetic analyses of insect Dorsal-like proteins and expression of up-stream factors. (A) No expression of Am-pipe mRNA is found in honeybee ovarioles. (B) Am-pipe mRNA is first detected throughout the posterior third of the stage 6 embryo (C) Am-cact mRNA is enriched to the dorsal side of the oocyte (stage 4 oocyte shown). (D) By the end of oogenesis (stage 9), Am-cact mRNA is enriched to the anterior pole of the oocyte. (E) Prior to gastrulation, Am-cact mRNA expression remains restricted to an anterior domain in the cellularised embryo. (F) Consensus Bayesian analysis tree for insect Dorsal-like proteins. Honeybee Dorsal-like proteins are indicated by shading. Numbers shown at the nodes are posterior probability values. (G) Am-GB19066 RNA is detected in the nurse cells and throughout the oocyte (mid-stage oocyte shown). (H) Am-GB19066 RNA is found throughout stage 4 embryos. (I) Expression of Am-GB18032 RNA is only detected in the posterior nurse cells during mid-oogenesis (asterisks). Abbreviations: Apis mellifera (Am), Drosophila melanogaster (Dm), Tribolium castaneum (TC), Bombus impatiens (Bimp), Nasonia vitripennis (Nv), Atta cephalotes (Acep), Acromyrmex echinatior (Aech), Camponotus floridanus (Cf), Bombyx mori (Bm), Delia antiqua (Da), Acyrthosiphon pisum (Ap), Nurse cell (NC), oocyte (o). All embryo images are oriented with anterior to the left and dorsal side up.

embryo the serosa membrane is retained (Figure 3C,D asterisks; nuclei are more sparsely distributed indicating serosa tissue), implying that the extraembryonic membranes have formed but, in the case of the serosa, have failed to expand to cover the embryo. Loss of function of Tribolium Toll results in the loss of DV asymmetry of the extra-embryonic tissue border, with retention of the amnion and serosa tissue [25].

$A m-D p p^{\mathrm{RNAi}}$ stage 9 embryos exhibited loss of anterior appendages, with loss of overt signs of segmentation (Figure $3 \mathrm{E}, \mathrm{F}$ ) and the tracheal pits are also enlarged
(Figure 4E). The amnion is reduced to cover a much smaller dorsal domain (arrows in Figure 3E). Loss of patterning is particularly obvious in the presumptive thoracic-anterior abdominal region (Figure 3E) and across the ventral side of the embryo there is a distinct groove (arrows Figure 3F) that appears to separate the two halves of the embryo.

Am-GB19066 knockdown results in a considerable broadening of the amnionic tissue (Figure 3G). The head appendages are present and appear normal (Figure 3G, $\mathrm{H})$. Thoracic abdominal and posterior tracheal pits are 

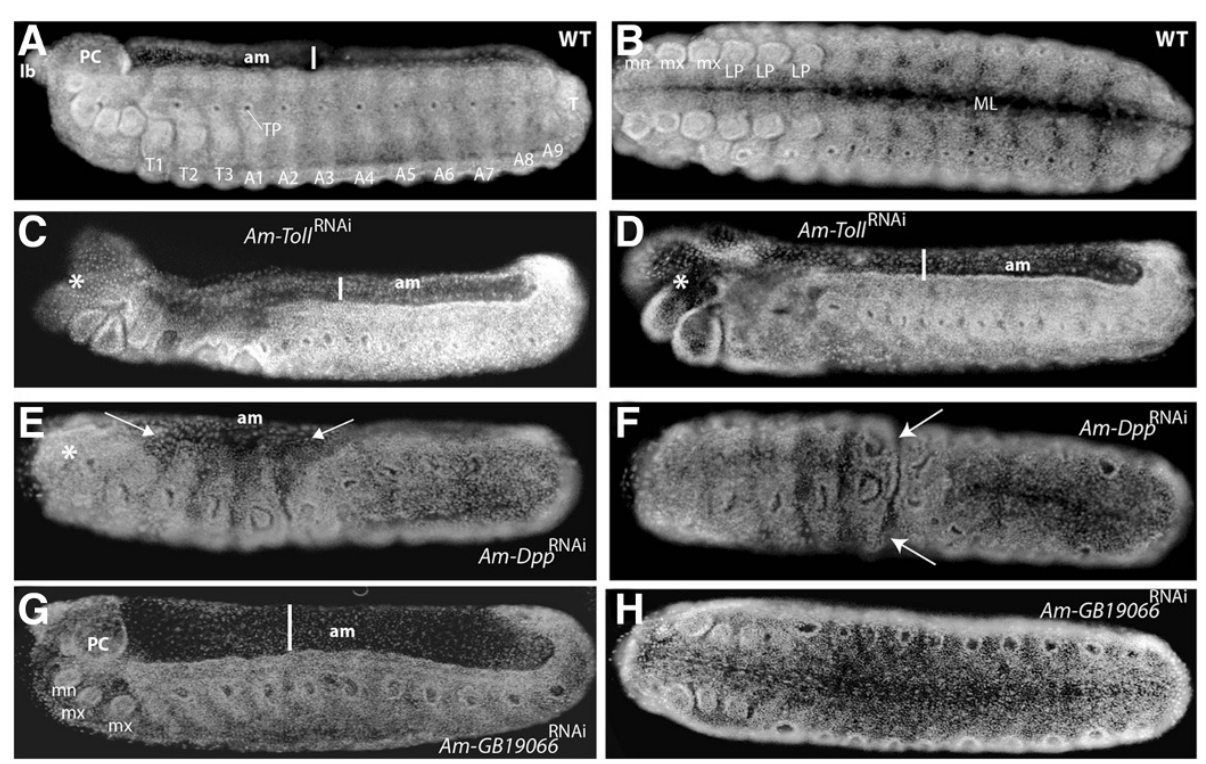

Figure 3 Embryos resulting from dsRNA-mediated knockdown of Am-Toll, Am-Dpp, and Am-GB19066. (A) DAPI stained wildtype (WT) embryo (stage 9, 48 h) with segments and appendages labelled. (B) Ventral view of a WT embryo at stage 9. (C) Stage 9 embryo following injection with dsRNA against Am-Toll. (D) A second example of typical stage 9 embryo after knockdown of Am-Toll, (E) Am-Dpp ${ }^{\mathrm{RNAi}}$, and (F) Am-Dpp dsRNA knockdown ventral side up. (G) and (H, ventral view) Am-GB19066 ${ }^{\mathrm{RNAi}}$. All embryo images are oriented with anterior to the left and dorsal side up unless stated otherwise. Abbreviations: midline (ML), amnion (am), procephalic lobe (PC), mandible (mn), maxillae (mx), limb pair (Ip), thorax (T), abdominal (A), terminal segment (T), tracheal pit (TP).

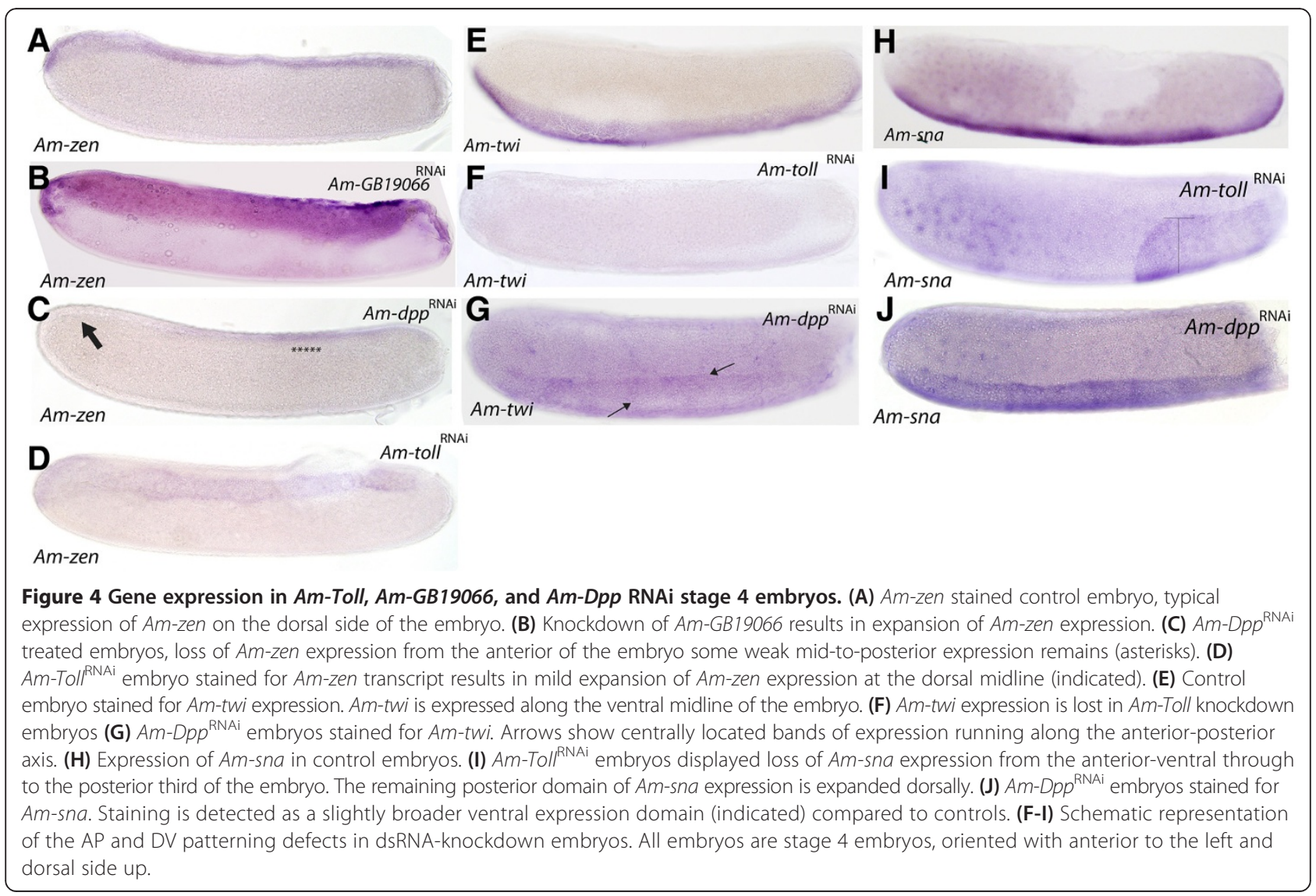


enlarged compared to wildtype (WT) embryos, and it is difficult to discriminate between body segments (compare Figure 3A to Figure 3G,H). These results imply that amnion patterning is disrupted with knockdown of a dorsal-like gene, with limited disorder of some ectoderm structures. This phenotype is milder than that of dorsal loss-of-function experiments in Drosophila, where mutants lack mesoderm and ventral ectoderm [52].

We also examined the expression patterns of three key genes regulated by the Toll and Dpp pathways in Drosophila in honeybee dsRNA knockdown embryos. Zen acts to pattern the extraembryonic membranes in Drosophila, honeybee, and Tribolium $[7,8,53]$. In honeybee embryos, Am-zen is expressed in several cells along the dorsal midline of the embryo and in an anterior-dorsal cap (Figure 4A) [7]. In GB-19066 ${ }^{\mathrm{RNAi}}$ embryos, Am-zen expression expanded to cover almost a third of the dorsal side of the embryo (Figure $4 \mathrm{~B}$ ), suggesting that the dorsal-like protein GB19066 has a role in restricted Dpp expression to a few cells at the dorsal-midline of the embryo. $A m-D p p^{\text {RNAi }}$ resulted in loss of $A m$-zen expression from the anterior-dorsal region of the embryo (Figure $5 \mathrm{C}$; the presumptive serosa [7]). Am-zen mRNA is still detected in a central to posterior-dorsal region of the $A m$ $D p p^{\mathrm{RNAi}}$ embryo (Figure $4 \mathrm{C}$, asterisks), typically where the amnion forms. Knockdown of Am-Toll resulted in an expansion of Am-zen expression across the dorsal midline (Figure 4E), in a domain almost double the number of cells of that seen in WT (Figure 4A).

The twist ( $t$ wi) gene, an immediate downstream target of the Toll pathway, is required for mesoderm specification, being expressed as a ventral domain in most insects (Figure 4E) [48]. In Tribolium, Tc-twi is found expressed as a ventral domain located in the posterior growth zone [25]. Am-twi expression is completely lost in $A m-$ Toll $^{\mathrm{RNAi}}$ embryos (Figure 4F). In $A m-D p p$
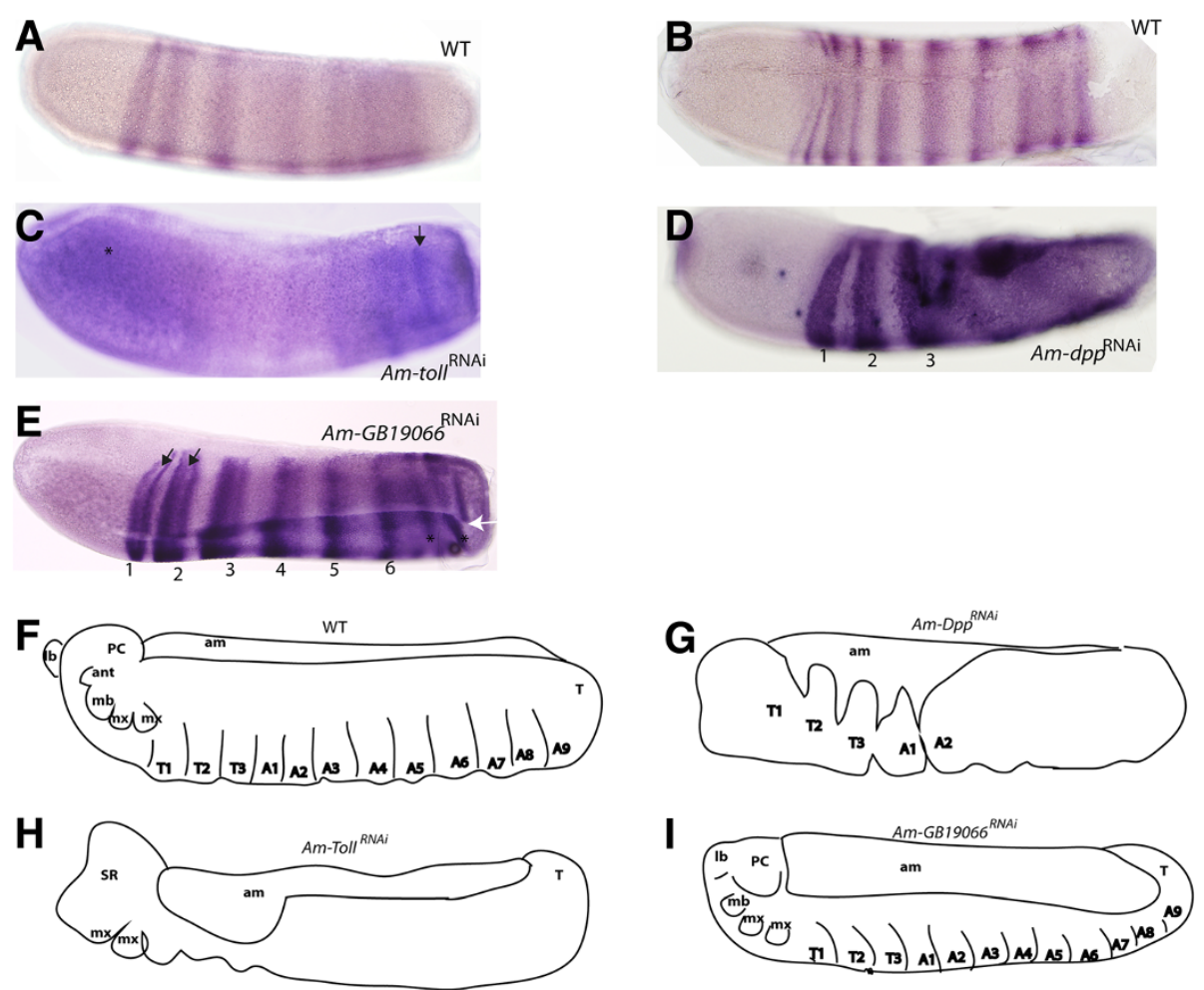

Figure 5 Am-eve expression in dsRNA knockdown embryos. (A and B) Am-eve expression at stages 3-4. Am-eve stripe expression starts at the anterior to posterior fashion. Stripes begin to split (arrow) and become distinguished just prior to gastrulation (B). (C) Am-eve mRNA is detected throughout stage 4 embryos following knockdown of Am-Toll. The strongest expression is found at the anterior and posterior ends of the embryo, and some stripe expression can be discerned (arrow). (D) In Am-Dpp ${ }^{\mathrm{RNAi}}$ embryos only the first three stripes (1-3) of Am-eve expression is seen. The posterior half of the embryo Am-eve mRNA appears as one broad domain of expression. (E) Am-eve transcripts are expressed in stripes after Am-GB19066 ${ }^{\mathrm{RNAi}}$ but are much less distinct, particularly throughout the posterior half of the embryo. The gastrulation ventral furrow appears expanded and there is some disruption of Am-eve stripes across this region (white arrow). F) Schematic representation of WT embryos at stage 9 with segments labeled. (G) Representation of the Am-Dpp ${ }^{\text {RNAi }}$ phenotype showing loss of most segments and amnion. (H) Summary schematic of $A m-T o / /^{\text {RNAi }}$ embryos labeling remaining segmens present in these embryos. (I) Representation of Am-GB19066 $6^{\mathrm{RNAi}}$ labeling remaining segments present in RNAi treated embryos. Abbreviations: amnion (am), procephalic lobe (PC), mandible (mb), maxillae (mx), thorax segment (T1-3), abdominal segment (A1-9), terminal segment (T), antenna (ant), labrum (Ib), serosa (SR). 
knockdown embryos, Am-twi mRNA is weakly detected thorough the entire ectoderm surface, although expression is stronger in a ventral domain (arrows, Figure 4G).

Snail expression is downstream of the Toll pathway and, as such, its expression in Drosophila and Tribolium is restricted to the ventral side of the embryo where it is required for gastrulation and neurogenesis [54-57]. This expression pattern is also conserved in honeybee embryos where Am-sna is expressed as a ventral stripe in blastoderm embryos (Figure 5H). In Am-Toll ${ }^{\mathrm{RNAi}}$ embryos, ventral Am-sna expression is lost from the anterior and abdominal ventral surface; however, the remaining posterior expression has expanded dorsally to form a wider band of expression (Figure 5I). In Am$D p p^{\mathrm{RNAi}}$ embryos, Am-sna is also expressed as a ventral stripe, although this region is significantly expanded (Figure 5J).

Since many 'DV pathway dsRNA' treated embryos showed AP patterning or segmentation defects, we also examined the expression of Am-even-skipped (Am-eve). Am-eve mRNA in honeybee is maternally expressed, and in early embryos is detected throughout the syncytial blastoderm and early cellularized embryo [58]. It later becomes refined to a broad abdominal domain before distinct stripes appear. At stage 4, Am-eve is expressed as seven broad stripes that later split into 14 stripes to establish the parasegment boundaries during segmentation (Figure 5A,B) [59]. Am-Toll ${ }^{\mathrm{RNAi}}$ results in disorganization of Am-eve expression at stage 4 (Figure 5C). Am-eve mRNA is found throughout the embryo with two possible distinct stripes found at the posterior end above the background of ubiquitous Am-eve expression (Figure 5C, arrows) and staining seems stronger at the anterior (Figure $5 \mathrm{C}$, asterisk). Loss of $A m-D p p$ expression results in $A m$-eve expression throughout the mid-posterior end of the embryo, although the first three stripes are still distinguishable (Figure 5D). Following knockdown of Am-GB1906, Am-eve stripes 1-6 are distinct and normal in appearance, with stripes one and two beginning to split (Figure 5E, arrows). However the posterior end stripes appear fragmented and disorganized across the DV axis (Figure 5E, asterisks).

Am-Toll and Am-Dpp have a greater influence on Am-eve expression (resulting in loss of the amnion and serosa tissues) than Am-zen, which has little effect on Am-eve stripe expression [7]. This implies the Dpp and Toll pathways influence the definition of the AP axis

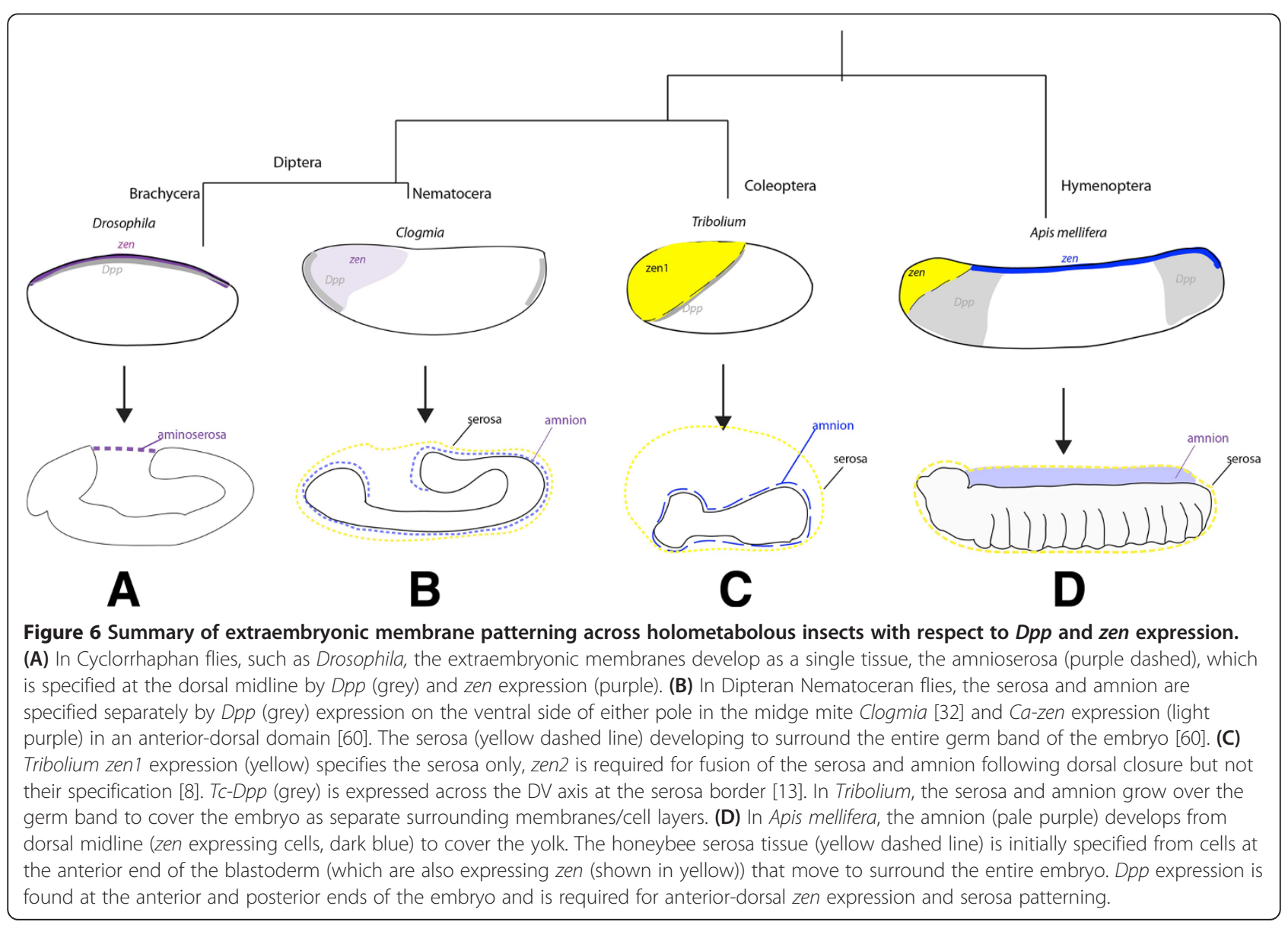


prior (Figure 5G,H) to establishment of Am-eve stripe expression during segmentation. This influence is in addition to their roles in DV axis establishment, and occurs independently of Am-zen.

\section{Conclusions}

This study set out to describe the expression and function of the Toll, Dorsal, and Dpp pathways in the long germ-band insect, $A$. mellifera, during early embryo development. Comparisons of the expression and function of individual components of the DV patterning pathways in insects reveal the roles of some factors are more highly conserved, particularly those acting later in these pathways. This is consistent with evolutionary changes in segmentation and axis formation in insects, where earlier acting components are evolving more rapidly than downstream factors $[7,27,36,61]$. For example sim, twist, and snail are expressed along the DV axis of the long and short germ-band embryos and a zen gene is expressed in regions where extraembryonic membranes will form. The most striking differences in spatial expression are found during early development. In honeybee embryos, the expression of many factors, including Am-Toll, Am-Dpp, and Am-cact, appears to be oriented with respect to the AP axis of the early embryo. This may be partially due to the way in which the extraembryonic membranes are determined in different insects (Figure 6). Establishing the position of the serosa-embryo border differs, for example in Tribolium (Figure 6B), where the amnion-embryo border is positioned asymmetrically across the AP axis [25], while in the honeybee the serosa initially patterns an anterior cap before moving posteriorly to enclose the embryo (Figure 6D). In Drosophila, aminoserosa tissue originates at the dorsal midline following zen expression (Figure 6A). These changes to the positioning of the extraembryonic membrane borders appear to require shifts in the spatial and temporal expression of Dpp and zen as described in Figure 6.

Our data implies that signalling pathways have considerable influence over cell fate determination across both axes. Loss of honeybee Toll and Dpp function not only resulted in loss of DV patterning but also disruption to patterning across the entire ectoderm. This suggests that patterning of both major body axes may be more codependent than previous studies in Drosophila have led us to understand.

\footnotetext{
Abbreviations

Am-eve: Am-even-skipped; Am-Toll/NAi: Am-Toll by dsRNAi; AP: Anteriorposterior; DV: Dorsal-ventral; Dpp: decapentaplegic; hb: hunchback; otd: orthodenticle; pip: pipe; RNAi: RNA interference; Sog: Short gastrulation; spz: spätzle; Tc-Dpp: Tribolium Dpp; tkv: thickveins; twi: twist; WT: Wildtype; zen: zenknüllt.
}

\section{Competing interests}

PKD is an editor of EvoDevo.

\section{Authors' contributions}

MJW conceived of the study, performed most of the experiments, and co-wrote the manuscript. PKD co-wrote the manuscript. NJK performed some of the cloning experiments and co-wrote the manuscript. All authors read and approved the final manuscript.

\section{Acknowledgments}

We would like to thank Otto Hyink for help with the honeybee culture and James Smith for feedback on manuscript drafts. Research was funded by a University of Otago grant to MJW and a Marsden grant to PKD (10-UOO-168).

\section{Author details}

${ }^{1}$ Laboratory for Evolution and Development, Genetics Otago and Gravida, Department of Biochemistry, University of Otago, P.O. Box 56, Dunedin 9054, New Zealand. ${ }^{2}$ Developmental Biology Laboratory, Department of Anatomy, University of Otago, P.O. Box 56, Dunedin 9054, New Zealand. ${ }^{3}$ Current address: Evolution and Development Research Group, Department of Zoology, University of Oxford, South Parks Road, Oxford OX1 3PS, UK.

Received: 7 October 2013 Accepted: 20 January 2014

Published: 12 March 2014

\section{References}

1. Lynch J, Desplan C: 'De-evolution' of Drosophila toward a more generic mode of axis patterning. Int J Dev Biol 2003, 47:497-503.

2. Lynch JA, El-Sherif E, Brown SJ: Comparisons of the embryonic development of Drosophila, Nasonia, and Tribolium. Dev Biol 2012, 1:16-39.

3. Rafiqi AM, Lemke S, Ferguson S, Stauber M, Schmidt-Ott U: Evolutionary origin of the amnioserosa in cyclorrhaphan flies correlates with spatial and temporal expression changes of zen. Proc Natl Acad Sci USA 2008, 105:234-239.

4. Panfilio KA, Liu PZ, Akam M, Kaufman TC: Oncopeltus fasciatus zen is essential for serosal tissue function in katatrepsis. Dev Biol 2006, 292:226-243.

5. Rafiqi AM, Park CH, Kwan CW, Lemke S, Schmidt-Ott U: BMP-dependent serosa and amnion specification in the scuttle fly Megaselia abdita. Development 2012, 139:3373-3382.

6. Dearden P, Grbic M, Falciani F, Akam M: Maternal expression and early zygotic regulation of the Hox3/zen gene in the grasshopper Schistocerca gregaria. Evol Dev 2000, 2:261-270.

7. Wilson MJ, Dearden PK: Diversity in insect axis formation: two orthodenticle genes and hunchback act in anterior patterning and influence dorsoventral organization in the honeybee (Apis mellifera). Development 2011, 138:3497-3507.

8. van der Zee M, Berns N, Roth S: Distinct functions of the Tribolium zerknullt genes in serosa specification and dorsal closure. Curr Biol 2005, 15:624-636.

9. Marques G, Musacchio M, Shimell MJ, Wunnenberg-Stapleton K, Cho KW, O'Connor MB: Production of a DPP activity gradient in the early Drosophila embryo through the opposing actions of the SOG and TLD proteins. Cell 1997, 91:417-426.

10. Ferguson EL, Anderson KV: Localized enhancement and repression of the activity of the TGF-beta family member, decapentaplegic, is necessary for dorsal-ventral pattern formation in the Drosophila embryo. Development 1992, 114:583-597.

11. Irish VF, Gelbart WM: The decapentaplegic gene is required for dorsalventral patterning of the Drosophila embryo. Genes Dev 1987, 1:868-879.

12. Sekelsky JJ, Newfeld SJ, Raftery LA, Chartoff EH, Gelbart WM: Genetic characterization and cloning of mothers against $\mathrm{dpp}$, a gene required for decapentaplegic function in Drosophila melanogaster. Genetics 1995, 139:1347-1358.

13. Van Der Zee M, Stockhammer O, Von Levetzow C, Nunes DA, Fonseca R, Roth S: Sog/Chordin is required for ventral-to-dorsal Dpp/BMP transport and head formation in a short germ insect. Proc Natl Acad Sci USA 2006, 103:16307-16312.

14. Wilson MJ, Abbott H, Dearden PK: The evolution of oocyte patterning in insects: multiple cell-signalling pathways are active during honeybee oogenesis and are likely to play a role in axis patterning. Evol Dev 2011, 13:127-137.

15. Lynch $J A$, Roth $\mathrm{S}$ : The evolution of dorsal-ventral patterning mechanisms in insects. Genes Dev 2011, 25:107-118. 
16. Peri F, Technau M, Roth S: Mechanisms of Gurken-dependent pipe regulation and the robustness of dorsoventral patterning in Drosophila. Development 2002, 129:2965-2975.

17. Moussian B, Roth S: Dorsoventral axis formation in the Drosophila embryo-shaping and transducing a morphogen gradient. Curr Biol 2005 15:R887-899.

18. Morgan MM, Mahowald AP: Multiple signalling pathways establish both the individuation and the polarity of the oocyte follicle in Drosophila. Arch Insect Biochem Physiol 1996, 33:211-230.

19. Dearden PK, Wilson MJ, Sablan L, Osborne PW, Havler M, McNaughton E, Kimura K, Milshina NV, Hasselmann M, Gempe T, Schioett M, Brown SJ, Elsik CG, Holland PW, Kadowaki T, Beye M: Patterns of conservation and change in honey bee developmental genes. Genome Res 2006, 16:1376-1384

20. Shigenobu S, Bickel RD, Brisson JA, Butts T, Chang CC, Christiaens O, Davis GK, Duncan EJ, Ferrier DE, Iga M, Janssen R, Lin GW, Lu HL, McGregor AP, Miura T, Smagghe G, Smith JM, van der Zee M, Velarde RA, Wilson MJ, Dearden PK, Stern DL: Comprehensive survey of developmental genes in the pea aphid, Acyrthosiphon pisum: frequent lineage-specific duplications and losses of developmental genes. Insect Mol Bio/ 2010, 19(Suppl 2):47-62.

21. Lynch JA, Peel AD, Drechsler A, Averof M, Roth S: EGF Signalling and the origin of axial polarity among the insects. Curr Biol 2010, 20(11):1042-1047.

22. Reach M, Galindo RL, Towb P, Allen JL, Karin M, Wasserman SA: A gradient of cactus protein degradation establishes dorsoventral polarity in the Drosophila embryo. Dev Biol 1996, 180:353-364

23. Belvin MP, Jin Y, Anderson KV: Cactus protein degradation mediates Drosophila dorsal-ventral signalling. Genes Dev 1995, 9:783-793.

24. Roth S, Hiromi Y, Godt D, Nusslein-Volhard C: Cactus, a maternal gene required for proper formation of the dorsoventral morphogen gradient in Drosophila embryos. Development 1991, 112:371-388.

25. Nunes DA, Fonseca R, Von Levetzow C, Kalscheuer P, Basal A, Van Der Zee $M$, Roth S: Self-regulatory circuits in dorsoventral axis formation of the short-germ beetle Tribolium castaneum. Dev Cell 2008, 14:605-615.

26. Finkelstein $\mathrm{R}$, Perrimon N: The orthodenticle gene is regulated by bicoid and torso and specifies Drosophila head development. Nature 1990, 346:485-488.

27. Lynch JA, Brent AE, Leaf DS, Pultz MA, Desplan C: Localized maternal orthodenticle patterns anterior and posterior in the long germ wasp Nasonia. Nature 2006, 439:728-732.

28. Pechmann M, McGregor AP, Schwager EE, Feitosa NM, Damen WG: Dynamic gene expression is required for anterior regionalization in a spider. Proc Natl Acad Sci U S A 2009, 106:1468-1472.

29. Kotkamp K, Klingler M, Schoppmeier M: Apparent role of Tribolium orthodenticle in anteroposterior blastoderm patterning largely reflects novel functions in dorsoventral axis formation and cell survival. Development 2010, 137:1853-1862.

30. Osborne PW, Dearden PK: Expression of Pax group III genes in the honeybee (Apis mellifera). Dev Genes Evol 2005, 215:499-508.

31. Ferguson EL: Conservation of dorsal-ventral patterning in arthropods and chordates. Curr Opin Genet Dev 1996, 6:424-431.

32. Munoz-Torres MC, Reese JT, Childers CP, Bennett AK, Sundaram JP, Childs KL, Anzola JM, Milshina N, Elsik CG: Hymenoptera Genome Database: integrated community resources for insect species of the order Hymenoptera. Nucleic Acids Res 2011, 39:D658-662.

33. Ronquist F, Huelsenbeck JP: MrBayes 3: Bayesian phylogenetic inference under mixed models. Bioinformatics 2003, 19:1572-1574.

34. Huson DH, Richter DC, Rausch C, Dezulian T, Franz M, Rupp R: Dendroscope: An interactive viewer for large phylogenetic trees. BMC Bioinforma 2007, 8:460.

35. Dearden PK, Duncan EJ, Wilson MJ: Whole-mount in situ hybridization of honeybee (Apis mellifera) tissues. Cold Spring Harb Protoc 2009, 2009(6):pdb. prot5225

36. Wilson MJ, Dearden PK: Tailless patterning functions are conserved in the honeybee even in the absence of Torso signalling. Dev Biol 2009, 335:276-287.

37. Dearden PK, Duncan EJ, Wilson MJ: RNA interference (RNAi) in honeybee (Apis mellifera) embryos. Cold Spring Harb Protoc 2009, 2009(6):pdb. prot5228.

38. Wotton KR, Alcaine Colet A, Jaeger J, Jimenez Guri E: Evolution and expression of BMP genes in flies. Dev Genes Evol 2013, 223(5):335-340.

39. St Johnston RD, Gelbart WM: Decapentaplegic transcripts are localized along the dorsal-ventral axis of the Drosophila embryo. Embo J 1987, 6:2785-2791.
40. Lemke S, Antonopoulos DA, Meyer F, Domanus MH, Schmidt-Ott U: BMP signalling components in embryonic transcriptomes of the hover fly Episyrphus balteatus (Syrphidae). BMC Genomics 2011, 12:278.

41. Goltsev Y, Fuse N, Frasch M, Zinzen RP, Lanzaro G, Levine M: Evolution of the dorsal-ventral patterning network in the mosquito, Anopheles gambiae. Development 2007, 134:2415-2424.

42. Dearden PK, Akam M: Early embryo patterning in the grasshopper, Schistocerca gregaria: wingless, decapentaplegic and caudal expression. Development 2001, 128:3435-3444.

43. Nellen D, Affolter M, Basler K: Receptor serine/threonine kinases implicated in the control of Drosophila body pattern by decapentaplegic. Cell 1994, 78:225-237.

44. Affolter M, Nellen D, Nussbaumer U, Basler K: Multiple requirements for the receptor serine/threonine kinase thick veins reveal novel functions of TGF beta homologs during Drosophila embryogenesis. Development 1994, 120:3105-3117.

45. O'Connor MB, Umulis D, Othmer HG, Blair SS: Shaping BMP morphogen gradients in the Drosophila embryo and pupal wing. Development 2006, 133:183-193.

46. Nunes DA, Fonseca R, Van Der Zee M, Roth S: Evolution of extracellular Dpp modulators in insects: The roles of Tolloid and twisted-gastrulation in dorsoventral patterning of the Tribolium embryo. Dev Biol 2010, 345:80-93.

47. Jazwinska A, Rushlow C, Roth S: The role of brinker in mediating the graded response to Dpp in early Drosophila embryos. Development 1999, 126:3323-3334.

48. Buchta T, Ozuak O, Stappert D, Roth S, Lynch JA: Patterning the dorsalventral axis of the wasp Nasonia vitripennis. Dev Biol 2013, 381:189-202.

49. Sen J, Goltz JS, Stevens L, Stein D: Spatially restricted expression of pipe in the Drosophila egg chamber defines embryonic dorsal-ventral polarity. Cell 1998, 95:471-481.

50. Blank V, Kourilsky P, Israel A: NF-kappa B and related proteins: Rel/dorsal homologies meet ankyrin-like repeats. Trends Biochem Sci 1992, 17:135-140

51. Steward R, Zusman SB, Huang LH, Schedl P: The dorsal protein is distributed in a gradient in early Drosophila embryos. Cell 1988, 55:487-495.

52. Anderson $\mathrm{KV}$, Nusslein-Volhard C: Information for the dorsal-ventral pattern of the Drosophila embryo is stored as maternal mRNA. Nature 1984, 311:223-227.

53. Doyle HJ, Harding K, Hoey T, Levine M: Transcripts encoded by a homoeo box gene are restricted to dorsal tissues of Drosophila embryos. Nature 1986, 323:76-79.

54. Sommer RJ, Tautz D: Expression patterns of twist and snail in Tribolium (Coleoptera) suggest a homologous formation of mesoderm in long and short germ band insects. Dev Genet 1994, 15:32-37.

55. Ashraf SI, IP YT: The Snail protein family regulates neuroblast expression of 56. inscuteable and string, genes involved in asymmetry and cell division in Drosophila. Development 2001, 128:4757-4767.

56. I $\mathrm{YT}$, Levine $\mathrm{M}$, Bier $\mathrm{E}$ : Neurogenic expression of snail is controlled by separable CNS and PNS promoter elements. Development 1994, 120:199-207.

57. Alberga A, Boulay JL, Kempe E, Dennefeld C, Haenlin M: The snail gene required for mesoderm formation in Drosophila is expressed dynamically in derivatives of all three germ layers. Development 1991, 111:983-992.

58. Wilson MJ, Dearden PK: Pair-rule gene orthologues have unexpected maternal roles in the honeybee (Apis mellifera). PLOS ONE 2012, 7:e46490.

59. Wilson MJ, Havler M, Dearden PK: Giant, Kruppel, and caudal act as gap genes with extensive roles in patterning the honeybee embryo. Dev Biol 2010, 339:200-211.

60. Stauber M, Prell A, Schmidt-Ott U: A single Hox3 gene with composite bicoid and zerknullt expression characteristics in non-Cyclorrhaphan flies. Proc Natl Acad Sci USA 2002, 99:274-279.

61. Olesnicky EC, Brent AE, Tonnes L, Walker M, Pultz MA, Leaf D, Desplan C: A caudal mRNA gradient controls posterior development in the wasp Nasonia. Development 2006, 133:3973-3982.

doi:10.1186/2041-9139-5-11

Cite this article as: Wilson et al:: Components of the dorsal-ventral pathway also contribute to anterior-posterior patterning in honeybee embryos (Apis mellifera). EvoDevo 2014 5:11. 